\title{
On Fuzzy Semi Extremely Disconnected in Fuzzy Topological Space
}

\author{
${ }^{1}$ Munir Abdul khalik Alkhafaji, ${ }^{2}$ Shymaa Abd Alhassan Alkanee \\ ${ }^{1,2}$ AL-Mustinsiryah University, College of Education, Department of Mathematics
}

\begin{abstract}
The aim of this paper to introduced a semi extremely disconnected space on Topological space in fuzzy sitting, Several properties and characterizations of such space are discussed.

Key words :- fuzzy semi extremely disconnected space, fuzzy semi hyper connected space, Generalized fuzzy semi extremely disconnected space.
\end{abstract}

\section{Introduction}

The term extremely disconnected was introduced by M.H. Stone [1].The concept of fuzzy set was introduced by Zadeh in his classical paper [2] in 1965 ,The fuzzy topological space was introduced by Chang [3] in 1968 , The fuzzy quasi coincident concept which introduced in 1980 by Pu and Lu [5], Azad [4] has introduced the concepts of fuzzy semi open set, fuzzy semi closed set.

\section{Preliminaries}

A fuzzy set $A$ in a universe set $X$ is characterization by a membership (characteristic) function $\mu_{\tilde{A}}: X$ $\rightarrow \mathrm{I}$, which an associated with each point $\mathrm{X}$ in $\mathrm{X}$ a real number in closed interval $\mathrm{I}=[0,1]$. The collection of all fuzzy subset in $\mathrm{X}$ will be denote by $\mathrm{I}^{\mathrm{X}}$ [2] A family T on fuzzy set $\mathrm{X}$ is called a fuzzy topology [3] on $\mathrm{X}$ if $\phi$ and $\mathrm{X}$ belong to $\mathrm{T}$ and $\mathrm{T}$ is closed with respect to arbitrary union and finite intersection. The members of $\mathrm{T}$ are called fuzzy open sets and their complements are fuzzy closed sets. In this paper we use the notion of a fuzzy topology in the original sense of Chang [3] We shall denote a fuzzy topological space(fts. for short) by (X, T), where $\mathrm{X}$ is the underlying set and $\mathrm{T}$ is the fuzzy topology .The symbols $\mu, \delta, \lambda \ldots$. etc. are used to denote fuzzy sets and the symbols $\mu(\mathrm{x}), \delta(\mathrm{x}), \lambda(\mathrm{x})$.. etc. are used to denote the membership function for this sets, the symbol $(1-\lambda)$ stands for the complement of the fuzzy set $\lambda$.

The interior and the closure of $\lambda$ in a fuzzy topological space (X, T) defned by Int $\lambda=U\{\delta: \delta \leq \lambda, \delta \in \mathrm{T}\}$, $\mathrm{Cl}=\cap\left\{\delta: \lambda \leq \delta, \delta \in T^{c}\right\}$ and denote by $\lambda^{0}, \bar{\lambda}$ respectively . A fuzzy point with singleton support $\mathrm{x} \in \mathrm{X}$ and the value $\mathrm{r}(0<\mathrm{r}<1)$ is denoted by $x_{r}$. In this paper we use the fuzzy topological space to introduced the fuzzy semi externally disconnected space and we give a necessary and sufficient condition for an fts to be semi extremely disconnected, proof some properties and relations, fuzzy semi hyper connected space and the relation with fuzzy semi extremely disconnected space and Generalized fuzzy semi extremely disconnected space and proof some theorems.

\section{1 - Basic definition}

Definition 1.1 [11] A fuzzy set $\mu$ of fts $(X, T)$ is said to be :-

1. Fuzzy semi open set if $: \mu(\mathrm{x}) \leq \operatorname{cl}(\operatorname{int}(\mu(\mathrm{x}))), \forall \mathrm{x} \in \mathrm{X}$.

2. Fuzzy semi closed set if $: \operatorname{int}(\operatorname{cl}(\mu(\mathrm{x}))) \leq \mu(\mathrm{x}), \forall \mathrm{x} \in \mathrm{X}$.

Definition 1.2 [11] Let $(X, T)$ be fts , The fuzzy semi interior and the fuzzy semi closure for a fuzzy set $\mu$ is defined by :-

1. $\mu_{0}=U\left\{\delta_{i}: \delta_{i} \in \mathrm{FSO}(\mathrm{X}), \delta_{i}(x) \leq \mu(\mathrm{x})\right\}, \forall \mathrm{x} \in \mathrm{X}$.

2. $\underline{\mu}=\cap\left\{\delta_{i}: \delta_{i}^{c} \in \mathrm{FSO}(\mathrm{X}), \mu(\mathrm{x}) \leq \delta_{i}(x)\right\}, \forall \mathrm{x} \in \mathrm{X}$.

where $\operatorname{FSO}(X, T)$ is the family of all fuzzy semi open set in fts $\mathrm{X}$. It is easy to verify the following relations between fuzzy semi interior and fuzzy semi closure :

(a) $1-\mu_{0}=\underline{1-\mu}$ (b) $1-\underline{\mu}=(1-\mu)_{0}$.

Proposition 1.3 [12] For any fuzzy set $\eta$ in fts (X, T) then :- $\eta^{0} \leq \eta_{0} \leq \eta \leq \bar{\eta} \leq \bar{\eta}$.

Deffinition 1.4 [9] A fuzzy point $x_{r}$ is said to be quasi coincident (overlap) with a fuzzy set $\mu$ in $\mathrm{X}$ denoted by $x_{r} \mathrm{q} \mu$ if $x_{r}+\mu(\mathrm{x})>1$ and $x_{r} \hat{q} \mu$ if $x_{r}+\mu(\mathrm{x})<1$.

Deffinition 1.5 [9] A fuzzy set $\delta$ is said to be quasi coincident with a fuzzy set $\mu$ in X denoted by $\delta \mathrm{q} \mu$ if there exists $\mathrm{x} \in \mathrm{X}$ such that $\delta(\mathrm{x})+\mu(\mathrm{x})>1$ and denote by $\delta \hat{q} \mu$ if $\delta(\mathrm{x})+\mu(\mathrm{x}) \leq 1, \forall \mathrm{x} \in \mathrm{X}$. 
Deffinition 1.6 [9][11] A fuzzy set $\delta$ in fuzzy topological space (X, T) is called fuzzy quasi neighborhood(fuzzy semi quasi neighborhood) of a fuzzy point $x_{r}$ in $\mathrm{X}$ if there exists a fuzzy open set (fuzzy semi open set $) \mu$ in X such that $x_{r} \mathrm{q} \mu$ and $\mu(\mathrm{x}) \leq \delta(\mathrm{x}), \forall \mathrm{x} \in \mathrm{X}$.

Deffinition $1.7[10]$ Let $(X, T)$ be any fuzzy topological space and A be any non-empty subset of $X$. define T/A $=\{\delta / \mathrm{A}: \delta \in \mathrm{T}\}$, then it is well known that T/A is a fuzzy topology in A and the fuzzy topological space (A, T/A) is called fuzzy subspace of $(X, T)$.

Proposition $1.8[13]$ Let $\left(\tilde{\mathrm{A}}, \tau^{\sim}\right)$ be fts. $\tilde{\mathrm{B}} \subseteq \tilde{\mathrm{A}}$ is a fuzzy set, $\tilde{\mathrm{G}} \subseteq \tilde{\mathrm{A}}$ is a fuzzy semi open set then $\tilde{\mathrm{B}} \hat{q} \tilde{\mathrm{G}} \Leftrightarrow \tilde{\mathrm{G}}$ $\hat{q} \operatorname{Scl}(\tilde{\mathrm{B}})$.

\section{2 - fuzzy semi extremely disconnected space}

Deffinition 2.1 A fuzzy topological space (X, T) is called fuzzy semi extremely disconnected space iff the semi closure of every fuzzy semi open set is a fuzzy semi open set i.e if $\mu \in \mathrm{FSO}(\mathrm{X}, \mathrm{T}) \rightarrow \underline{\mu} \in \mathrm{FSO}(\mathrm{X}, \mathrm{T})$.

Example 2.2 Let $\mathrm{X}$ be a non empty set and $\mathrm{T}=\{\phi, \mathrm{X}\} \cup\left\{\mu_{\alpha}\right\}, \alpha \in \vartheta$ where $\mu_{\alpha}$ is any fuzzy set defined on $\mathrm{X}$ such that $\frac{3}{4}<\mu_{\alpha}(\mathrm{x})<1$, for every $\mathrm{X}$ in $\mathrm{X}$. Then the fts $(\mathrm{X}, \mathrm{T})$ is fuzzy semi extremely disconnected .

Theorem 2.3 A fuzzy topological space $(X, T)$ is fuzzy semi extremely disconnected iff for every pair of nonoverlaping fuzzy semi open sets $\mu$ and $\delta$ in $\mathrm{X}, \mu$ and $\underline{\delta}$ are non - overlapping fuzzy sets in X.

Proof :- Let $\mu$ and $\delta$ is a fuzzy semi open sets in a fuzzy semi extremely disconnected space $(\mathrm{X}, \mathrm{T})$ such that $\mu \hat{q}$ $\delta$ by proposition (1.8) $\rightarrow \underline{\mu} \hat{q} \delta$ since $(\mathrm{X}, \mathrm{T})$ is a fuzzy semi extremely disconnected space $\underline{\mu}$ is a fuzzy semi open set hence by proposition $\overline{(1.8)} \underline{\mu} \hat{q} \underline{\delta}$

Conversely:- Let $\mu$ be a fuzzy semi open set in $\mathrm{X} \mu$ and $1-\mu$ are fuzzy semi open sets in $\mathrm{X}$ such that $\mu \hat{q}(1$ - $\underline{\mu})$ by hypothesis $\underline{\mu} \hat{q} \underline{(1-\underline{\mu})} \rightarrow \underline{\mu}(x)+\underline{(1-\underline{\mu})}(x) \leq 1$, for all $\mathrm{X}$ in $\mathrm{X} \rightarrow \underline{\mu}(x) \leq 1-$ $\left.(1-\underline{\mu})(x)=1-\left(\underline{\left(\mu^{c}\right)_{0}}\right)(x)=1-\underline{\left(1-\mu_{0}\right)(x)=1-(1-(\underline{\mu})} 0\right)(x)=(\underline{\mu})_{0}$. for all $\mathrm{x}$ in $\mathrm{X}$.

$\rightarrow \mu$ is a fuzzy semi open set in $X$.Then the space $(X, T)$ is a fuzzy semi extremely disconnected .

Theorem 2.4 Every open subspace of semi extremely disconnected fts is a semi extremely disconnected.

Proof :- Clear .

Theorem 2.5 For any fuzzy topological space the following statement are equivalent :-

(1) $(X, T)$ is a fuzzy semi extremely disconnected .

(2) For each fuzzy semi closed set $\lambda, \lambda_{0}$ is a fuzzy semi closed set.

(3) For each fuzzy semi open set $\lambda$ we have $\underline{\lambda}+(1-\underline{\lambda})=1$.

(4) For every pair of fuzzy semi open sets , $\mu$ in $X$ with $\underline{\lambda}+\mu=1$ we have $\underline{\lambda}+\mu=1$.

Proof $(\mathbf{1}) \rightarrow \overline{(2)}$ Let $\lambda$ be a fuzzy semi closed set in a fuzzy semi extremely disconnected space $(\mathrm{X}, \mathrm{T}) \rightarrow$ $\lambda^{c}$ is a fuzzy semi open set $\underline{\lambda^{c}}$ is a fuzzy semi open set $\rightarrow\left(\underline{\left(\lambda^{c}\right.}\right)^{c}$ is a fuzzy semi closed set but $\left(\lambda^{c}\right)^{c}=\lambda_{0}$ $\rightarrow \lambda_{0}$ is a fuzzy semi closed set.

Proof (2) $\rightarrow(\mathbf{3})$ Let $\lambda$ is a fuzzy semi open set, since $(\underline{\lambda})^{c}=\left(\lambda^{c}\right)_{0}$

$\underline{\lambda}+(1-\underline{\lambda})=\underline{\lambda}+\underline{\left(\lambda^{c}\right)_{0}}=\underline{\lambda}+\left(\lambda^{c}\right)_{0}($ by $(2))$

$=\underline{\lambda}+\overline{(\underline{\lambda})^{c}=} \underline{\lambda}+(1-(\underline{\lambda}))=1$.

Proof $(3) \rightarrow(4)$ Let $\lambda, \mu$ are fuzzy semi open sets in $(\mathrm{X}, \mathrm{T})$ such that $\underline{\lambda}+\mu=1$....(1*)

Then by (3), $\underline{\lambda}+\underline{(1-\underline{\lambda})}=1=\underline{\lambda}+\mu \rightarrow \mu=(1-\underline{\lambda}) \ldots . .\left(2^{*}\right)$ but from $\left(1^{*}\right), \mu=1-\underline{\lambda}$ and so from (2*) $1-\underline{\lambda}=\overline{(1-\underline{\lambda})} \rightarrow 1-\underline{\lambda} \in \mathrm{FSO}(\mathrm{X})$ and so $\underline{\mu}=1-\underline{\lambda}$, That is $\underline{\mu}+\underline{\lambda}=1$.

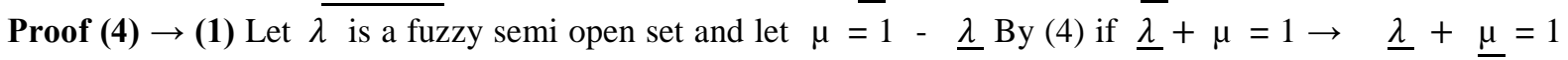
$\ldots .(*)$, To show $\underline{\lambda}$ is a fuzzy semi open set or To show $\left(\lambda^{c}\right)_{0}$ is a fuzzy semi closed set.by $(*)$ we get $\underline{\mu}=1$ $\underline{\lambda}=\left(\lambda^{c}\right)_{0}$ since $\underline{\mu}$ is a fuzzy semi closed set $\rightarrow\left(\lambda^{c}\right)_{0}$ is a fuzzy semi closed set $\rightarrow \underline{\lambda}$ is a fuzzy semi open set. Then the fts $(\bar{X}, \mathrm{~T})$ is semi extremely disconnected space.

Theorem 2.6 A fuzzy topological space $(\mathrm{X}, \mathrm{T})$ is a fuzzy semi extremely disconnected space if and only if $\underline{\mu}=(\underline{\mu})_{0}$ for every fuzzy semi open set $\mu$ in $X$.

Proof :- Let $\mu$ is a fuzzy semi open set in a fuzzy semi extremely disconnected space $\quad(X, T) \rightarrow \underline{\mu}$ is a fuzzy semi open set $\rightarrow \underline{\mu}=(\underline{\mu})_{0}$.

Conversely if $\mu$ is a fuzzy semi open set $\rightarrow \underline{\mu}=(\underline{\mu})_{0}$, hence $\underline{\mu}$ is a fuzzy semi open set $\rightarrow(\mathrm{X}, \mathrm{T})$ is a fuzzy semi extremely disconnected space. 
Theorem 2.7 A fuzzy topological space (X, T) is a fuzzy semi extremely disconnected space if and only if $\mu_{0}=\mu_{0}$ for every fuzzy semi closed set $\mu$ in $X$.

Proof :- Let $\mu$ is a fuzzy semi closed set in a fuzzy semi extremely disconnected space

$(\mathrm{X}, \mathrm{T}) \rightarrow \mu^{c} \quad$ is a fuzzy semi open set and $\underline{\mu^{c}}=\left(\underline{\mu^{c}}\right)_{0} \rightarrow\left(\underline{\mu^{c}}\right)^{c}=\left(\left(\underline{\mu^{c}}\right)_{0}\right)^{c} \rightarrow \mu_{\mathbf{0}}=\underline{\mu_{0}}$

Conversely : Let $\mu$ is a fuzzy semi open set $\rightarrow \mu^{c}$ is a fuzzy semi closed set in $\mathrm{X}$ and by hypothesis we get $\left(\mu^{c}\right)_{0}=\left(\underline{\left(\mu_{0}^{c}\right.}\right)$ and $\left(\left(\mu^{c}\right)_{0}\right)^{c}=\left(\underline{\left(\mu_{0}^{c}\right)}\right)^{c}$

hence $\mu=(\mu)_{0} \rightarrow(\mathrm{X}, \mathrm{T})$ is a fuzzy semi extremely disconnected space.

Proposition 2.8 Every extremely disconnected fts is a semi extremely disconnected fts but not conversely as shown in the following example.

Proof :- Trivial.

Example 2.9 Let $X=\{a, b, c, d\}, T=\left\{\phi, \mu_{1}, \mu_{2}, \mu_{3}, X\right\}, \mu_{1}=\{(a, 0.7),(b, 0.7)\}, \mu_{2}=\{(d, 0.7)\}, \mu_{3}=$ $\{(\mathrm{a}, 0.7),(\mathrm{b}, 0.7),(\mathrm{d}, 0.7)\}, T^{c}=\left\{\phi, \mu_{1}{ }^{c}, \mu_{2}{ }^{c}, \mu_{3}{ }^{c}, \mathrm{X}\right\}, \quad \operatorname{FSO}(\mathrm{X}, \mathrm{T})=\left\{\phi, \mu_{1}, \mu_{2}, \mu_{3}, \mu_{4}, \mu_{5}, \mathrm{X}\right\}, \mu_{4}$ $=\{(\mathrm{a}, 0.7),(\mathrm{b}, 0.7),(\mathrm{c}, 0.7)\}, \mu_{5}=\{(\mathrm{c}, 0.7),(\mathrm{d}, 0.7)\}$, since $\underline{\mu} \in \mathrm{FSO}(\mathrm{X}, \mathrm{T}), \forall \mu \in \mathrm{FSO}(\mathrm{X}, \mathrm{T})$ but $\bar{\mu}=\mu_{4}$ is not fuzzy open set.

\section{3 - fuzzy semi hyper connected space}

Deffinition 3.1 A space $X$ is said to be fuzzy semi hyper-connected if the intersection of two non-empty fuzzy semi open sets is non-empty. i.e $\forall \mu, \lambda \in \operatorname{FSO}(\mathrm{X}, \mathrm{T})$ then $\mu \cap \lambda \neq \phi$ or $\underline{\mu}=\mathrm{X}, \forall \mu \in \mathrm{FSO}(\mathrm{X}, \mathrm{T})$.

Theorem 3.2 Every semi hyper connected fts is a semi extremely disconnected

fts but not conversely as shown in the example 2.9

Proof :- Trivial.

Theorem 3.3 Every open subspace of a fuzzy semi hyper connected space is fuzzy semi hyper connected.

Proof :- Trivial.

\section{4 - Generalized fuzzy semi extremely disconnected space}

Deffinition 4.1 [11] A fuzzy set $\mu$ in fts $(X, T)$ is said to be generalized Fuzzy semi open set (gfs - open) if for each fuzzy semi closed set $\delta$ in $X$ such that $\delta \leq \mu \rightarrow \delta \leq \operatorname{sint}(\mu)$.

Deffinition 4.2 [11] A fuzzy set $\mu$ in fts $(X, T)$ is said to be generalized Fuzzy semi closed set (gfs - closed) if for each fuzzy semi open set $\delta$ in $X$ such that $\mu \leq \delta \rightarrow \operatorname{scl}(\mu) \leq \delta$.

Deffinition 4.3 [11] Let $(X, T)$ be fts,$\mu \subseteq X$, The fuzzy semi interior ${ }^{*}$ for a fuzzy set $\mu$ is defined by :$\operatorname{sint}^{*}(\mu)=U\left\{\delta_{i}: \delta_{i} \in \mathrm{FGSO}(\mathrm{X}, \mathrm{T}), \delta_{i}(\mathrm{x}) \leq \mu(\mathrm{x})\right\}, \forall \mathrm{x} \in \mathrm{X}$.

where $\operatorname{FGSO}(X, T)$ is the family of all fuzzy generalized semi open set.

Deffinition $4.4[11]$ Let $(\mathrm{X}, \mathrm{T})$ be fts,$\mu \subseteq \mathrm{X}$, The fuzzy semi closure $e^{*}$ for a fuzzy set $\mu$ is defined by :$s c l^{*}(\mu)=\cap\left\{\delta_{i}: \delta_{i}{ }^{c} \in \mathrm{FGSO}(\mathrm{X}, \mathrm{T}), \mu(\mathrm{x}) \leq \delta_{i}(\mathrm{x})\right\}, \forall \mathrm{x} \in \mathrm{X}$.

Deffinition 4.5 The fts $(X, T)$ is said to be Generalized fuzzy semi extremely disconnected space if $s c l^{*}(\mu)$ is a gfs - open set, for every gfs - open set $\mu$.

Theorem 4.6 For any fuzzy topological space $(X, T)$, the following are equivalent .

[1] $(\mathrm{X}, \mathrm{T})$ is generalized fuzzy extremely disconnected space .

[2] For each gfs- closed set, $\operatorname{sint}^{*}(\lambda)$ is a gfs - closed set.

[3] For each gfs - open set $\lambda$ we have $s c l^{*}(\lambda)+s c l^{*}\left(1-s c l^{*}(\lambda)\right)=1$.

[4] For every pair of gfs - open sets $\lambda, \mu$ in X with $s c l^{*}(\lambda)+\mu=1$

we have $s c l^{*}(\lambda)+s c l^{*}(\mu)=1$.

Proof $(\mathbf{1}) \rightarrow(\mathbf{2})$ Let $(\mathrm{X}, \mathrm{T})$ is generalized fuzzy extremely disconnected space and $\lambda$ is a gfs - closed set , To show $\operatorname{sint}^{*}(\lambda)$ is a gfs - closed set, we claim $\lambda$ is a gfs - closed set $\rightarrow 1-\lambda$ is a gfs - open set and since 1 $\operatorname{sint}^{*}(\lambda)=\operatorname{scl}^{*}\left(\lambda^{c}\right)$ and by (1) $1-\operatorname{sint}^{*}(\lambda)$ is a gfs - open set $\rightarrow \operatorname{sint}^{*}((\lambda))$ is a gfs - closed set .

Proof (2) $\rightarrow(3)$ since $s c l^{*}(\lambda)+s c l^{*}\left(1-s c l^{*}(\lambda)\right)=s c l^{*}(\lambda)+s c l^{*}\left(\operatorname{sint} t^{*}(1-\lambda)\right.$ and $\lambda$ is a gfs - open set $\rightarrow 1-\lambda$ is a gfs - closed set, by (2) $\operatorname{sint}^{*}(1-\lambda)$ is a gfs - closed set $\rightarrow \operatorname{scl}^{*}\left(\operatorname{sint}^{*}(1-\lambda)\right)=\operatorname{sint}^{*}(1-\lambda)$. Thus we get $s c l^{*}(\lambda)+s c l^{*}\left(1-s c l^{*}(\lambda)\right)=s c l^{*}(\lambda)+\operatorname{sint}^{*}(1-\lambda)=s c l^{*}(\lambda)+1-s c l^{*}(\lambda)=1$.

Proof (3) $\rightarrow$ (4) Let $\lambda, \mu$ be any gfs - open sets such that $s c l^{*}(\lambda)+\mu=1 \ldots \ldots$ (2*) by (3) $s c l^{*}(\lambda)+s c l^{*}(1$ - $\left.s c l^{*}(\lambda)\right)=1=s c l^{*}(\lambda)+\mu \rightarrow \mu=s c l^{*}\left(1-s c l^{*}(\lambda)\right) \ldots \ldots\left(3^{*}\right)$ but from $\left(2^{*}\right) \mu=1-s c l^{*}(\lambda) \rightarrow 1-s c l^{*}$ $\left.(\lambda)=s c l^{*}\left(1-s c l^{*} \lambda\right)\right) \ldots . .\left(4^{*}\right)$ from $\left(3^{*}\right)$ and $\left(4^{*}\right) \mu=s c l^{*}(\mu) \ldots .\left(5^{*}\right)$ put it in $\left(2^{*}\right) \rightarrow s c l^{*}(\lambda)+s c l^{*}(\mu)=1$. Proof (4) $\rightarrow$ (1) Let $\lambda$ is a gfs - open set in X and $\mu=1-\operatorname{scl}^{*}(\lambda)$, by (4) $\operatorname{scl}^{*}(\mu)=1-\operatorname{scl}^{*}(\lambda)=\operatorname{sint}^{*}\left(\lambda^{c}\right)$ since $\operatorname{scl}^{*}(\mu)$ is a gfs - closed set $\rightarrow \operatorname{sint}^{*}\left(\lambda^{c}\right)$ is a gfs - closed set $\rightarrow s c l^{*}(\lambda)$ is a gfs - open set .

\section{References}

[1] M.H. Stone, Algebraic characterizations of special Boolean rings , Fund. Math., 29 (1937), 223 - 302. 
[2] L. A. Zadeh, Fuzzy sets, Inform. and Control 8 (1965), 338 - 353.

[3] C. L. Chang, Fuzzy topological spaces ,J. Math. Anal. Appl. 24 (1968), 182 - 190

[4] K.K. Azad, On fuzzy semi-continuity,fuzzy almost cointinuity and fuzzy weakly continuity, J. Math.Anal.Appl.82,14 - 32 (1981).

[5] Pao-Ming, P. and Ying-Ming, L. Fuzzy Topology .I. Neighborhood Structure of a Fuzzy Point and Moore-Smith Convergence, J. Math. Anal. Appl.,ol.76, pp.571-599, (1980).

[6] N. Bourbaki, General Topology, Part I, Addison Wesley, Reading, Mass. 1996.

[7] Ratna Dev Sarma, Extermally Disconnected in Fuzzy Topological Space, MATEMATIQKI VESNIK , 46 (1994), 17 - 23.

[8] M. Caldas, G. Navalagi, and R. Saraf, On fuzzy weakly semiopen functions, Proyec ciones 21 (2002), 51 - 63 .

[9] Z. Petri_cevi_c, On S-Closed and Extermally Disconnected fuzzy topological space, MATEMATIQKI VESNIK, 50 (1998), 37 - 45

[10] G. Balasubramanian and V. Chandrasekar, Fuzzy $\alpha$ - Connected and Fuzzy $\alpha$ - Disconnected in Fuzzy Topological Space , MATEMATIQKI VESNIK , 56 (2004), $47-56$

[11] F.S.Mahmoud, M.A.Fath Alla, and S.M.Abd Ellah, . Fuzzy topology on fuzzy set : fuzzy semi continuity and fuzzy semiseparation axioms , Applied mathematics and computation (2003)

[12] S. Ganguly and S. Saha, A note on semi open sets in fuzzy topological space, fuzzy sets and system 18 (1986), 83 - 96 .

[13] A . A. Nouh, On convergence theory in fuzzy topological spaces and its applications , J . Dml. Cz. Math , 55(2)(2005), 295-316. 